\title{
FPGA-Based Implementation of the Back-EMF Symmetric-Threshold-Tracking Sensorless Commutation Method for Brushless DC-Machines
}

\author{
Araz Darba, Student Member, IEEE, Frederik De Belie, Member, IEEE, Aboubakr Salem, \\ Jan Melkebeek, Senior Member, IEEE
}

\begin{abstract}
The operation of brushless DC permanent-magnet machines requires information of the rotor position to steer the semiconductor switches of the power-supply module which is commonly referred to as Brushless Commutation. Different sensorless techniques have been proposed to estimate the rotor position using current and voltage measurements of the machine. Detection of the back-electromotive force (EMF) zero-crossing moments is one of the methods most used to achieve sensorless control by predicting the commutation moments. Most of the techniques based on this phenomenon have the inherit disadvantage of an indirect detection of commutation moments. This is the result of the commutation moment occurring 30 electrical degrees after the zero-crossing of the induced back-emf in the unexcited phase. Often, the time difference between the zero crossing of the back-emf and the optimal current commutation is assumed constant. This assumption can be valid for steadystate operation, however a varying time difference should be taken into account during transient operation of the BLDC machine. This uncertainty degrades the performance of the drive during transients. To overcome this problem which improves the performance while keeping the simplicity of the back-emf zero-crossing detection method an enhancement is proposed. The proposed sensorless method operates parameterless in a way it uses none of the brushless dc-machine parameters. In this paper different aspects of experimental implementation of the new method as well as various aspects of the FPGA programming are discussed. Proposed control method is implemented within a Xilinx Spartan 3E XC3S500E board.
\end{abstract}

Index Terms-Permanent-magnet brushless DC-machine (BLDC-machine), back-EMF zero-crossing, sensorless control, field-programmable gate arrays (FPGAs)

\section{INTRODUCTION}

$\mathbf{P}$ ERMANENT-MAGNET machines are widely used because of their high power density and efficiency. They are often classified in two topologies based on the wave form of the produced back-emf. The first class is supplied with sinusoidal voltages and currents and generates a back-emf which is assumed to be mainly sinusoidal. This category is often referred to as Permanent-Magnet Synchronous Machines (PMSM) or Brushless AC-machines (BLAC-machine). The other class is referred to as Brushless DC-machines (BLDC-machine). The supply current of these

Copyright (c) 2013 IEEE. Personal use of this material is permitted However, permission to use this material for any other purposes must be obtained from the IEEE by sending a request to pubs-permissions@ieee.org.

A. Darba "Email: araz.darba@ugent.be", F. De Belie "Email: frederik.debelie@ugent.be" and J. Melkebeek "Email: jan.melkebeek@ugent.be" are with the Department of Electrical Energy, Systems and Automation of Ghent University, St-Pietersnieuwstraat 41, B-9000 Gent, Belgium. machines has a rectangular shape and the wave form of the back-emf is approximately trapezoidal. The advantage of a BLDC-machine to a PMSM is that driving a BLDC-machine requires less components and processor efforts than a PMSM. Moreover, more power is produced by the BLDC-machine for a given peak value of current and back-emf due to the square waveform of the current. To drive a PMSM in an optimal way, the rotor-position information should be known with high resolution (several electrical degrees resolution) but in the case of a BLDC-machine, a sixty-degrees resolution sensor is sufficient. Nevertheless, the accuracy of the sensor should be within the range of several electrical degrees for both topologies.

Steering signals of the inverter switches use the rotorposition information of the machine to control the current flowing through the phases of the BLDC-machine. To extract the rotor position information of a permanent-magnet machine an auxiliary instrument such as optical rotary encoder or a resolver or hall-effect sensors might be used but the trend in modern and cost-effective drives is to replace the noise sensitive and less reliable mechanical sensors by numerical algorithms, often referred to sensorless or self-sensing methods. The advantage of these methods is the use of current or voltage measurements often available as they are required for the control of the power electronics or the protection of the inverter. Avoiding the position sensor yields remarkable savings in production costs, installation and maintenance and improves reliability. A wide variety of different sensorless methods has been proposed by different authors for a wide range of operation speeds from standstill to high speeds [1]-[3]. In [1], [2] a high-frequency test-signal injection is used to estimate the position of the rotor in such a way the average-current samples are unaffected by the test signals for BLDC and PMSM machines. In fact, the machine itself is used as a sensor. Therefore these methods are often referred as self-sensing methods rather than sensorless methods. In [3] motor voltages and currents are measured to estimate the flux position. These techniques can be used in many drives such as for PMSM as well as BLDC-machine drives. Other techniques are developed specifically for BLDC-machine drives. These methods can be fitted in one of the following categories: detection of the back-emf zero-crossing, back-emf integration, stator third-harmonic voltage component, detection of the conduction state of the 
free-wheeling diode. The back-emf is induced in the stator windings by the rotation of the permanent-magnets on the rotor. Therefore it depends on the rotor position as well as the rotor speed. By using this dependency it is possible to estimate the position and speed of the rotor by measuring and processing the back-emf. An overview of back-emf based sensorless methods for BLDC-machine drives can be found in [4]. In the back-emf zero-crossing detection the unenergized phase voltage is sensed with respect to the virtual neutral point. In [5], it has been implemented on a microprocessor and a low-pass filter is used to remove the high frequency components. The delay of the low-pass filter has a constant value for different speeds and limits the speed range. In [6], [7] a new back-emf zero-crossing detection is proposed by using the negative terminal voltage of the dc inverter supply instead of neutral point of the machine but extracting the back-emf signal of the un-excited phase after a zero-crossing moment is not implemented. Integration of the back-emf in the unenergized phase is a way to detect the back-emf zero-crossing moment as discussed in [8]. This method is less sensitive towards switching noise but it has some offset errors arising from the integration action. If the motor phase windings are star connected and the star point is available for measurements, summation of the three phase voltages results in a third harmonic voltage. Integration of the third harmonic voltage results in the third harmonic flux of the motor. Zero-crossing moments of the third harmonic flux correspond to the commutation moments [9]. This method shows the exact moment of commutation but the noise-to-signal ratio in the lower speed range is more critical compared to other methods because of the relatively low amplitude of the third harmonic. And the access to the neutral point of the motor is necessary which is not always available. Another indirect method to detect the zero-crossing is obtained by using the conduction state of the free-wheeling diode as is proposed in [10]. This method works well in a wide range of speed but it requires a lot of electronic circuits to detect the current of the free-wheeling diodes. In [11] the commutation signals are directly detected from the average line-to-line voltages obtained from filtered PWM waveforms. In [12] the terminal voltages are measured with respect to the negative dc-link voltage and used to determine the zero-crossing moment of the back-emf but obtaining the exact moment of switching was done by adding 30 degree delay to the zero-crossing moment of the back-emf.

In [13], the authors have proposed a method which after a predefined small delay from the last switching event that can be generated by start-up sequence, a voltage sample of the unenergized phase is obtained. By using this sampled value a threshold is defined for the back-emf of the same phase. As soon as the back-emf crosses the threshold added with the same delay which was applied before, the commutation action is triggered. Simulation results in [13] verified the performance of this method in detecting the exact moment of commutation which affects the transient states of the drive. In this paper different experimental aspects of implementation of the proposed method will be discussed and the results will

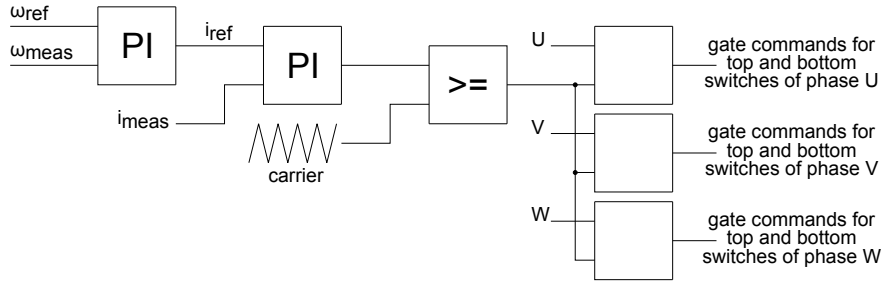

Fig. 1. Speed and current controllers

be illustrated.

New trends in industry as well as academic area are more towards replacing micro-controllers with newer generation of processing units which are FPGA's aimed to reach the optimum point of cost and technology. This objective pushes us to use a device that is able of doing many parallel calculations at the same time. Parallelism and reuse of resources features of FPGAs provide the demand of massive amount of calculations in short period of time. Examples of implementation of complex methods are in model-based predictive control [14], [15] or hardware-in-loop platforms [16]. Compared to DSPs and micro-controllers, FPGAs offer two dominant advantages: firstly, computational speeds are much faster by using parallel computational structures, secondly, the freedom to customize the individual signal and coefficient formats can be exploited for overcoming the numerical difficulties caused by finiteword-length effects. Which means, the possibility to obtain a higher precision of data formats at critical points in the calculation and a smaller precision at other points which is fixed in DSPs or micro-controller. In this paper a Xilinx Spar$\tan 3 \mathrm{E}$ XC3S500E evaluation board is used for implementing the proposed sensorless method as well as speed and current controllers, obtaining measurements and data acquisition.

\section{SPEED AND CURRENT CONTROLler}

A PI speed controller is designed to control the speed of the machine. Speed reference and indirect measurement of the speed are the inputs of the speed controller. Inputs of the PI-controller are sampled synchronously with commutation pulses. The current is measured by using only a single shunt resistor in the dc-link, reducing the cost and requiring a minimum amount of electronics to process the measurements. A PWM PI-controller with the carrier frequency of $10 \mathrm{kHz}$ keeps the dc-link current within the limits of the reference current. The inputs of the PI current controller are reference and measured dc-link currents. They are sampled at the carrier frequency. Fig. 1 shows the basic concept of speed and current controllers. The current of each phase is determined by sampling the dc-link current. The value of the sampled current is assigned to the energized phases of the motor with respect to the direction of the current in each conduction interval. The direction of the current for each energized phase is determined by the commutation sequence of the motor. By using this method the phase current control is possible by controlling only the dc-link current. 

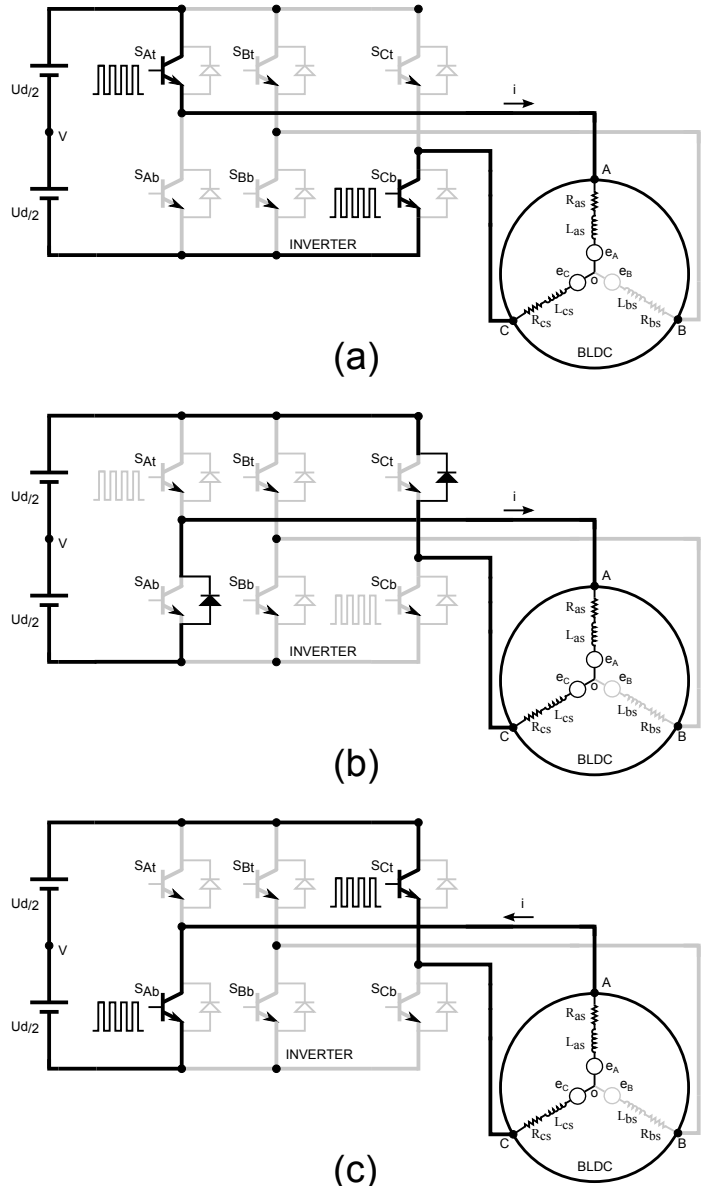

(c)

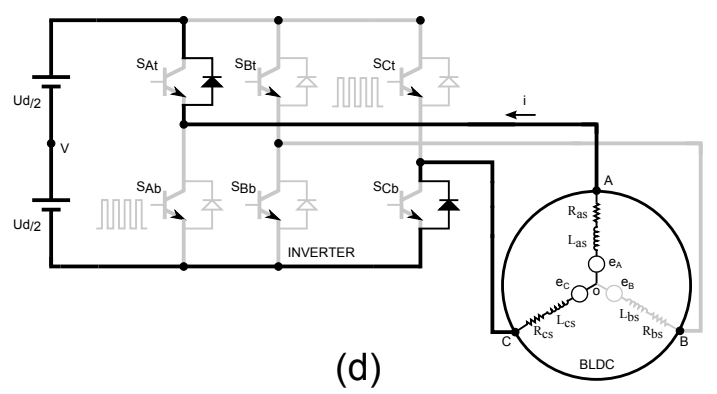

Fig. 2. Switching strategy: (a,b) motor mode, $(\mathrm{c}, \mathrm{d})$ generator mode. Control pulses for the deactivated switches are shown in grey

To control the dc-link current in the motor mode working condition as shown in Fig. 2-a, By closing SAt, SCb switches in the same time $+\mathrm{Vdc}$ voltage by SAt switch is applied to the phase A of the machine and current loop is closed by SCb. No complementary PWM pulses are applied to the other switches. In this switching strategy, unlike conventional switching strategies for BLDC machines the bottom switches of three inverter legs during their conduction interval aren't always kept ON. Instead, the correspondent lower switch is chopped with the same command signal as upper switch of the other leg of inverter (for example in Fig. 2-a, SAt and $\mathrm{SCb}$ have same driving signals). When the PWM signal turns to low the current path is closed as shown in Fig. 2-b and the current flow is handled by diodes.

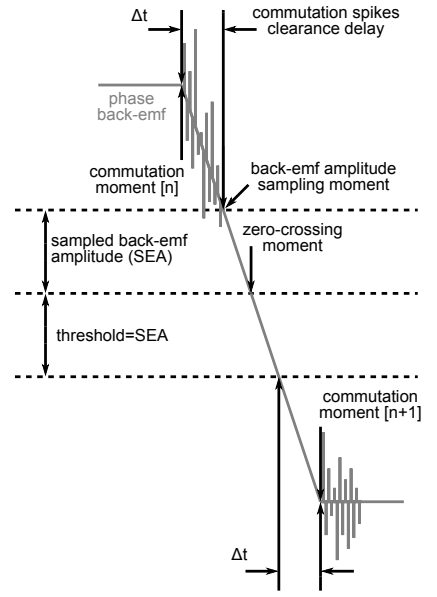

Fig. 3. Representation of the proposed method timings and events

For the regenerative working mode conditions (reverse current), current flow is transferred from SAt to SAb and in the leg $\mathrm{C}$, is transferred from $\mathrm{SCb}$ transistor to $\mathrm{SCt}$. For the moments that the PWM signal is high, the current loop is as shown in Fig. 2-c. For the moments that the PWM signal is low SAb, SCt are turned $\mathrm{OFF}$ and the current is flowing through the diodes which is illustrated in Fig. 2-d.

\section{Sensorless Control Using BACK-EMF SYMMETRIC THRESHOLD TRACKING}

The method used in this paper, as described by the authors in [13], is based on the sampling of the unenergized phase back-emf voltage and monitoring of the same phase back-emf voltage until it reaches a certain threshold, as shown in Fig. 3. This threshold is computed by taking the negative of the sampled back-emf voltage amplitude just after a commutation event [n] with a time difference of $\Delta t$. A $\Delta t$ delay has to be applied after each commutation moment to let the stochastic spikes of the commutation action which would add important noise to the measurements be cleared. This small clearance delay $\Delta t$ as shown in Fig. 3 is after the commutation event [n] before sampling the back-emf amplitude. The value of the $\Delta t$ is stored in a register to be used later. The sign of this sampled back-emf voltage value is changed and the resulting voltage is stored in a register as well. The value of this register which is called threshold register is then compared to the varying back-emf voltage. Some time later, as soon as the back-emf voltage crosses the value of the threshold register a delay equal to $\Delta t$ is applied and after this delay a commutation pulse is introduced to the controller and inverter.

\section{Open-loop Start-up Sequence}

The output signals and working principles of the openloop start-up ramp sequence are designed to be compatible with the sensorless control part. The proposed sensorless method is based on generating pulses which determine the 
optimal commutation moments of the BLDC machine. There are two different important pulsing signals in our system: processor clock pulse which is fixed to the $50 \mathrm{MHz}$ clock frequency and the commutation pulse signal which has a variable frequency and dependent on the speed of the machine. Commutation pulses are also used to update the back-emf and the speed indirect measurements as well as sampling the PI speed controller inputs. First step in the design of the open-loop start-up sequence is to determine the acceleration rate. The limiting factor for the acceleration rate depends on the start-up current of the machine and capability of the supply to provide enough current. To set a new open-loop start-up with higher acceleration rates or under-load start-up these two constraints should be taken into account. Aligning of the rotor position is done by considering few hundred milliseconds delay between the first and second pulse of the start-up sequence. By energizing two phases of the machine for a short time interval, $d q$ axis of the rotor are aligned with the $d q$ axis of the stator flux. This time must be determined with respect to the inertia of the machine. This alignment method is only applicable for such applications which the initial position of the machine isn't important. In most applications, it is acceptable if the motor turns slightly in either direction before starting. To implement the open-loop start-up, a sequence of commutation pulses are generated in a way that the time between two successive pulses decreases. The value of the time decrease between commutation pulses determines the rate of acceleration.

\section{Implementation of Sensorless Control Method}

The theoretical basis of our method is explained in [13] and Section III of this paper. First step in the implementation of this algorithm is collecting the back-emf information of the machine. This method requires a continuous information of machine back-emf during unexcited interval of each phase. By over-sampling the terminal voltage of each phase during its unenergized interval and by merging the obtained signals from each phase together a continuous signal is generated. In this paper this signal is called $E_{\text {inf }}(t)$ which has the useful backemf information of all three phases of the machine. $E_{\text {inf }}(t)$ signal is generated in the FPGA as:

$$
E_{\text {inf }}(t)=V_{T}(t)-V_{N}(t)
$$

where $V_{T}(t)$ is the sampled terminal voltage of the machine during its unenergized interval and $V_{N}(t)$ is the neutral voltage or virtual neutral voltage of the machine. Both of $V_{T}(t)$ and $V_{N}(t)$ are referenced to the negative side of the dclink voltage. $E_{\text {inf }}(t)$ has the unenergized phase back-emf information of the machine in each moment and is used to define the threshold. Determining the value of the threshold is explained in [13] and section VI of this paper as well. Fig. 4 shows the measured $E_{\text {inf }}(t)$ which is obtained from Xilinx ChipScope ${ }^{\mathrm{TM}}$ Pro. The ChipScope ${ }^{\mathrm{TM}}$ Pro tools integrate key logic analyser and other test and measurement hardware components with the target design inside the FPGA. While a design is running on the FPGA, user can trigger when certain events take place and view any of your design's internal

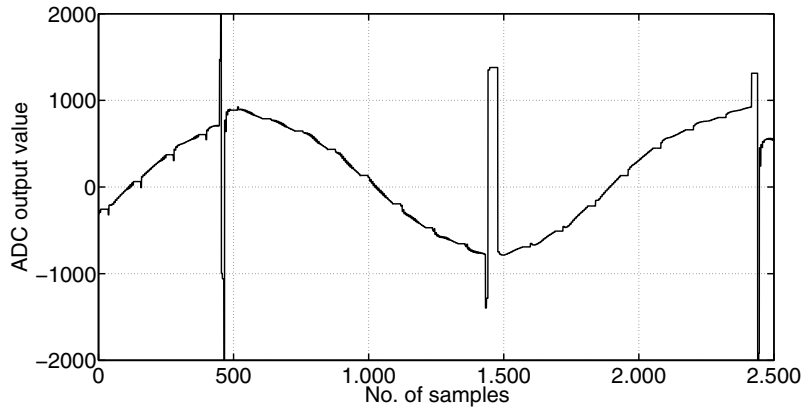

Fig. 4. $E_{\text {inf }}(t)$ measured results, operation condition: speed 1140 r.p.m, $50 \%$ nominal load, closed loop sensorless with current control

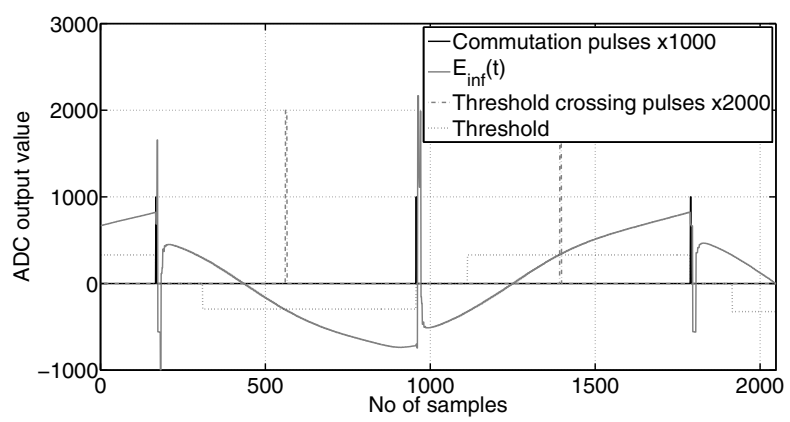

Fig. 5. Experimental results, operation condition: speed 1140 r.p.m, no-load, closed loop sensorless with current control

signals. The large spikes which are visible in Fig. 4 are due to the commutation in the phases of the machine and changing the source of $E_{\text {inf }}(t)$ from one phase to another. These spikes has no influence on our algorithms because they are ignored by parameter $\Delta t$ which is explained in section III. On the other hand, $E_{\text {inf }}(t)$ is monitored to detect its crossing moment from a defined threshold. Fig. 5 shows the experimental results from a drive system which is operated by the proposed sensorless algorithm. The parameters of the BLDC machine used in this study are presented in appendix A. In this figure the threshold, $E_{\text {inf }}(t)$, the detected threshold crossing moments and the commutation moments are illustrated which are also obtained from Xilinx ChipScope ${ }^{\mathrm{TM}}$ Pro.

\section{DAtA ACQUisitions AND MEAsurements}

In [13] the simulations have been done by assuming that the back-emf information of the machine is available. The back-emf was generated by using analytical equations of the machine. In this paper the back-emf information of the machine is extracted from sampling of the terminal voltages of the machine. This requires good interaction between control strategy, power electronics and machine. To implement this sensorless method, the back-emf voltage of the unenergized phase is sampled during 60 degrees for a specific phase chosen by a finite-state machine. For this purpose three analog-to-digital converters (ADC) are used to sample each phase voltage of the machine. In addition this sensorless method needs to receive the information of the neutral point voltage of the machine which it is sampled by 


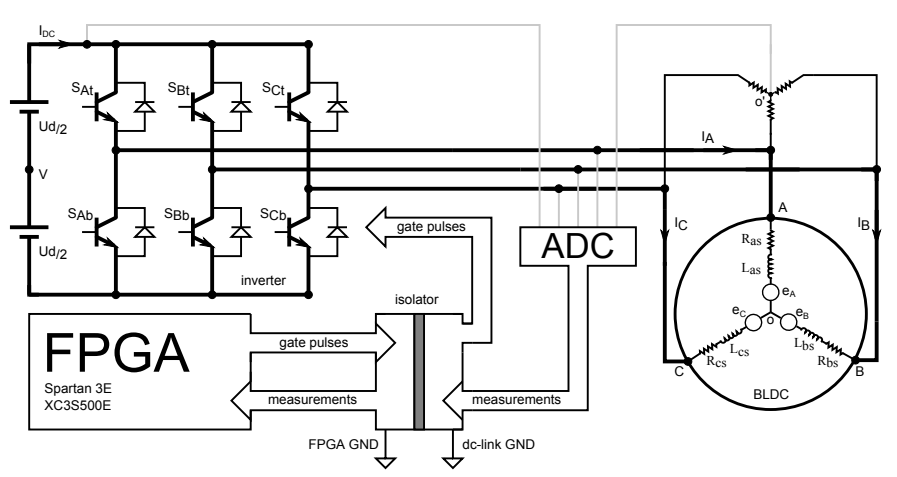

Fig. 6. Schematic diagram of the test setup

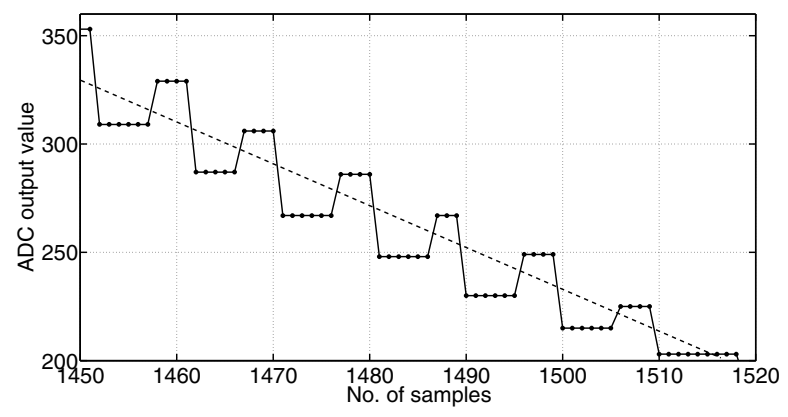

Fig. 7. Zoomed area for digitalized back-emf measurement, dotted line is the average value of samples and bullets are the sampled values

another ADC channel. For the machine used in this study the neutral point wire isn't available so that a virtual neutral point is implemented by using three resistors as shown in Fig. 6. Another ADC channel is used to sample the dc-link current. All acquired analog data from the machine after conversion to the digital data are isolated from power side by using IL175 four-channel digital isolators before being used by FPGA. Control commands and steering signals of the switches are also isolated in the same way before being applied to the power switches. The digital isolation scheme is shown in Fig. 6.

To define an accurate value for the threshold, the output of the ADC needs to be filtered. Fig. 7 shows the output of an ADC which is converting analog back-emf measured value to a digital signal. By averaging few neighbour data, more realistic results for the value of the back-emf is obtainable. For this purpose, five preceding samples and five subsequent samples of the back-emf signal together with the sample at the exact moment of sampling are averaged. Due to the fact that, the value of threshold will be used after the back-emf zero-crossing moment, there is sufficient time to process the sampled values and the delay of averaging doesn't have any effect on performance of the drive. By using this method an accurate value for threshold is obtained without having the disadvantages of adding analog filter before conversion to digital signal. A 210 us time period of the back-emf digital signal is shown in Fig. 7 with the sampling frequency of 3 us. ADCs are synchronised to

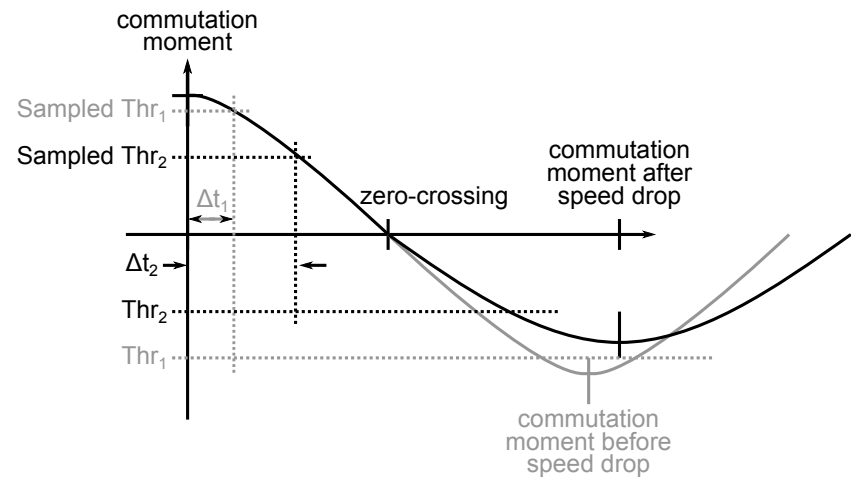

Fig. 8. Changing of the $\Delta t$ and Threshold values due to a reduction in the speed

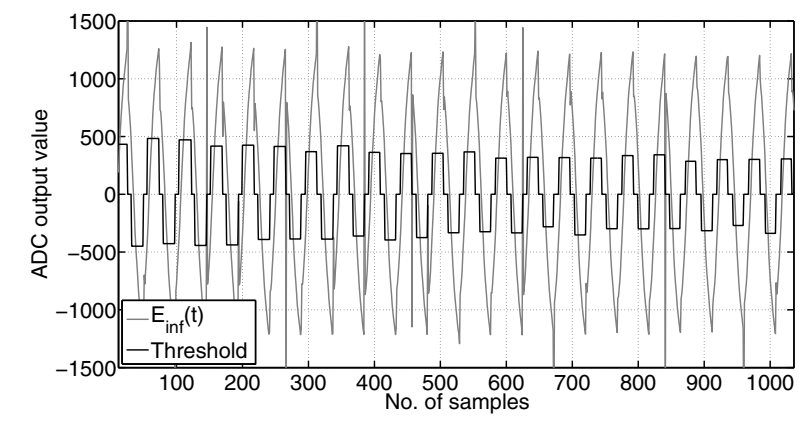

Fig. 9. Loading of the machine, nominal load, 2500 r.p.m

sample between PWM pulses to eliminate the switching noises. The switching actions result in high frequency noises in the virtual neutral point voltage and this has a direct effect on the sampled back-emf voltage of the unenergized phase. Speed and back-emf values are indirectly measured by counting the number of clock periods between two successive commutation moments. A detailed explanation about this method and the equations are published in [13] by the authors.

The value of the $\Delta t$ needs to be adapted with respect to the different working conditions of the machine. Due to a decrease in the speed of the machine during the loading conditions or change of the speed reference, the value of the threshold needs to be reduced to guarantee the crossing of the back-emf value from the sampled back-emf threshold after speed reduction. This situation is illustrated in Fig. 8 which the black solid waveform (which represents the amplitude of the back-emf after a drop in speed of the machine) doesn't cross the dashed grey line $T h r_{1}$ (which was defined as SampledThr 1 before a speed reduction after a $\Delta t_{1}$ delay after the first commutation moment). To solve this issue, a new delay $\Delta t_{2}$ is considered to define a new threshold value SampledTh $h_{2}$ which is lower then SampledTh $h_{1}$. The procedure of defining $\Delta t$ is explained in [13]. The measurement results for loading transients of the machine is illustrated in Fig. 9. It can be seen that, due to the reduction in the speed of the machine after applying a load torque the value of the threshold is reduced by sensorless algorithm. 


\section{CONCLUSION}

Conventional back-emf zero-crossing based sensorless commutation methods, are widely used in different industrial and low-power application with their own drawbacks. An enhanced sensorless commutation method based on backemf symmetric threshold-tracking method is proposed in this paper. Simulation results verify that satisfactory performance can be achieved with the proposed sensorless commutation method. Compared to the conventional commutation methods, the proposed method could have some advantages such as: better performance in systems with high transients, higher speed, control performance increases with speed as the backemf increases.

\section{APPENDIX A}

PARAMETERS OF THE TESTED MACHINE

\begin{tabular}{|c|c|}
\hline Parameter & Value \\
\hline \hline Stator Resistance & $0.170 \Omega$ \\
\hline Stator Inductance Constant & $0.28 e^{-3} H$ \\
\hline Number of Pole Pairs & 12 \\
\hline Nominal Voltage & $18 \mathrm{~V}$ \\
\hline Nominal Current & $19.4 \mathrm{~A}$ \\
\hline Nominal Power & $350 \mathrm{~W}$ \\
\hline
\end{tabular}

\section{ACKNOWLEDGMENT}

This work was supported by the Interuniversity Attraction Pole under Project (IUAP) P7/02. The work of A. Darba was supported by a project from the Special Research Fund (BOF) of UGent.

\section{REFERENCES}

[1] F. De Belie, J. De Backer, A. Darba, J. Melkebeek, "Low-Speed SalientPole BLDC-Machine Control by Using a Single Sensor," 15th IEEE International Conference on Electrical Machines and Systems (ICEMS), vol., no., pp.1,6, 21-24 Oct. 2012.

[2] F. De Belie, P. Sergeant and J. Melkebeek "A Sensorless Drive by Applying Test Pulses Without Affecting the Average-Current Samples" IEEE Transactions Power Electronics., Vol. 24 (4), pp. 875-888, April 2010 .

[3] S.B. Ozturk, H.A. Toliyat, "Direct Torque and Indirect Flux Control of Brushless DC Motor," IEEE/ASME Transactions on Mechatronics, vol.16, no.2, pp.351-360, April 2011

[4] P. Acarnley and J. Watson, "Review of Position-Sensorless Operation of Brushless Permanent-Magnet Machines," IEEE Transactions on Industrial Electronics, Vol. 53(2), pp.352-362, April 2006.

[5] Iizuka Kenichi, Uzuhashi Hideo, Kano Minoru, Endo Tsunehiro, Mohri Katsuo, "Microcomputer Control for Sensorless Brushless Motor," IEEE Transactions on Industry Applications, vol.IA-21, no.3, pp.595-601, May 1985.

[6] Jianwen Shao, D. Nolan, M. Teissier, D. Swanson, "A novel microcontroller-based sensorless brushless DC (BLDC) motor drive for automotive fuel pumps," IEEE Transactions on Industry Applications, vol.39, no.6, pp.1734-1740, Nov./Dec. 2003.

[7] Jianwen Shao, "An Improved Microcontroller-Based Sensorless Brushless DC (BLDC) Motor Drive for Automotive Applications," IEEE Transactions on Industry Applications, vol.42, no.5, pp.1216,1221, Sept./Oct. 2006.

[8] R.C. Becerra, T.M. Jahns, M. Ehsani, "Four-quadrant sensorless brushless ECM drive," IEEE Conference Proceedings, Sixth Annual Applied Power Electronics Conference and Exposition, 1991, (APEC '91), vol., no., pp.202-209, 10-15 Mar. 1991.

[9] J.C. Moreira, "Indirect sensing for rotor flux position of permanent mag net AC motors operating over a wide speed range," IEEE Transactions on Industry Applications, vol.32, no.6, pp.1394-1401, Nov./Dec. 1996.
[10] S. Ogasawara, H. Akagi, "An approach to position sensorless drive for brushless DC motors," IEEE Transactions on Industry Applications, vol.27, no.5, pp.928-933, Sep./Oct. 1991.

[11] Cheng-Hu Chen, Ming-Yang Cheng, "A New Cost Effective Sensorless Commutation Method for Brushless DC Motors Without Phase Shift Circuit and Neutral Voltage," IEEE Transactions on Power Electronics, vol.22, no.2, pp.644-653, March 2007.

[12] P. Damodharan, K. Vasudevan, "Sensorless Brushless DC Motor Drive Based on the Zero-Crossing Detection of Back Electromotive Force (EMF) From the Line Voltage Difference," IEEE Transactions on Energy Conversion, vol.25, no.3, pp.661-668, Sept. 2010.

[13] A. Darba, F. De Belie, J. Melkebeek, "Sensorless commutation and speed control of Brushless DC-machine drives based on the back-EMF symmetric threshold-tracking," IEEE International Electric Machines Drives Conference (IEMDC), vol., no., pp.492,497, 12-15 May 2013.

[14] T. J. Vyncke, S. Thielemans, M. Jacxsens, and J. A. Melkebeek, "Analysis of some design choices in model based predictive control of flying-capacitor inverters," COMPEL: The International Journal for Computation and Mathematics in Electrical and Electronic Engineering, vol. 31, no. 2, p. 619-635, Feb. 2012

[15] S. Thielemans, T. J. Vyncke, and J. A. Melkebeek, "Weight factor selection for model based predictive control of a four-level flyingcapacitor inverter," IET Power Electron., vol 5, no. 3, p. 323-333, March 2012.

[16] A. Darba, F. De Belie, T. Vyncke, J. Melkebeek, "FPGA-based realtime simulation of sensorless control of PMSM drive at standstill," IEEE International Symposium on Power Electronics, Electrical Drives, Automation and Motion (SPEEDAM), vol., no., pp.1063,1068, 20-22 June 2012. 